\title{
Crystal Structure of the Toll/Interleukin-1 Receptor Domain of Human IL-1RAPL*
}

Received for publication, March 29, 2004, and in revised form, April 26, 2004 Published, JBC Papers in Press, April 30, 2004, DOI 10.1074/jbc.M403434200

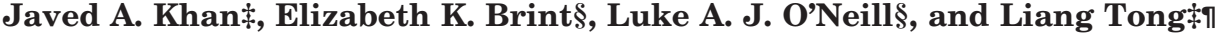 \\ From the $\ddagger$ Department of Biological Sciences, Columbia University, New York, New York 10027 and the §Cytokine \\ Research Group, Department of Biochemistry, Trinity College, Dublin 2, Ireland
}

The Toll/interleukin-1 receptor (TIR) domain is conserved in the intracellular regions of Toll-like receptors (TLRs) and interleukin-1 receptors (IL-1Rs) as well as in several cytoplasmic adapter molecules. This domain has crucial roles in signal transduction by these receptors for host immune response. Here we report the crystal structure at 2.3- $\AA$ resolution of the TIR domain of human IL-1RAPL, the first structure of a TIR domain of the IL-1R superfamily. There are large structural differences between this TIR domain and that of TLR1 and TLR2. Helix $\alpha \mathrm{D}$ in IL-1RAPL is almost perpendicular to its equivalent in TLR1 or TLR2. The BB loop contains a hydrogen bond unique to IL-1RAPL between Thr residues at the 8th and 10th positions. The structural and sequence diversity among these domains may be important for specificity in the signal transduction by these receptors. A dimer of the TIR domain of IL-1RAPL is observed in the crystal, although this domain is monomeric in solution. Residues in the dimer interface are mostly unique to IL-1RAPL, which is consistent with the distinct functional roles of this receptor. Our functional studies show IL-1RAPL can activate JNK but not the ERK or the p38 MAP kinases, whereas its close homolog, TIGIRR, cannot activate JNK. Deletion mutagenesis studies show that the activation of JNK by IL-1RAPL does not depend on the integrity of its TIR domain, suggesting a distinct mechanism of signaling through this receptor.

Toll-like receptors (TLRs) ${ }^{1}$ and interleukin-1 receptors (IL$1 R s)$ have crucial roles in host immune and inflammatory responses (1). A total of $10 \mathrm{TLRs}$ have been identified from the human genome. They recognize conserved molecular patterns in pathogenic microbial organisms, and this recognition is indispensable for the activation of the innate immune systems as well as the adaptive immune systems in vertebrates $(2,3)$. Ten

* This work was supported by National Institutes of Health Grant AI49475 (to L. T.). The costs of publication of this article were defrayed in part by the payment of page charges. This article must therefore be hereby marked "advertisement" in accordance with 18 U.S.C. Section 1734 solely to indicate this fact.

The atomic coordinates and structure factors (code 1T3G) have been deposited in the Protein Data Bank, Research Collaboratory for Structural Bioinformatics, Rutgers University, New Brunswick, NJ (http://www.rcsb.org/).

ๆ To whom correspondence should be addressed. Tel.: 212-854-5203; Fax: 212-854-5207; E-mail: tong@como.bio.columbia.edu.

${ }^{1}$ The abbreviations used are: TLR, Toll-like receptors; TIR, Toll/ interleukin-1 receptor; IL-1R, interleukin-1 receptor; JNK, c-Jun $\mathrm{NH}_{2}$ terminal kinase; ERK, extracellular signal-regulated kinase; MAP, mitogen-activated protein; TIGIRR, three immunoglobulin domaincontaining IL-1 receptor-related; IL-1RAPL, IL-1R accessory proteinlike; r.m.s., root mean square; MAD, multi-wavelength anomalous diffraction. different IL-1Rs are present in the human genome. IL-1R type I (IL-1RI) and IL-1R accessory protein (IL-1RAcP) mediate the pleiotropic effects of the proinflammatory cytokine IL-1, whereas IL-18R and AcPL function as receptors for the IL-18 cytokine (4). As their names imply, IL-1RI and IL-18R have high affinities for their ligands, whereas the accessory proteins (IL-1RAcP and AcPL) cannot bind the ligand directly.

The TLRs and IL-1Rs have entirely different extracellular domains. The TLRs contain many copies of the leucine-rich repeat (LRR), whereas the IL-1Rs contain three copies of the Ig-like domains. However, these two families of receptors share a conserved intracellular domain, known as the Toll/interleukin-1 receptor (TIR) domain. This domain is also present in several cytoplasmic molecules, such as MyD88, TIRAP/MAL, TRIF/TICAM, and TIRP/TRAM, which function as signal adapters for these receptors (5).

TIR domains contain 150-200 amino acid residues, and their sequences are weakly conserved among the TLRs, IL-1Rs, and the adapter molecules (Fig. 1). The amino acid sequence identity between any pair of TIR domains is generally about $25 \%$. The authenticity and integrity of this domain is crucial for the functions of these receptors. For example, a single-site mutation (Pro-712 $\rightarrow$ His, also known as the Lps ${ }^{\mathrm{d}}$ mutation) in the TIR domain of TLR4 renders mice unresponsive to lipopolysaccharide, the cell wall component of Gram-negative bacteria (6). This Pro residue is conserved in many of the TIR domains (Fig. 1 ), and the mutation of this residue to His in these other proteins generally produces dominant negative molecules.

IL-1RAPL (IL-1R accessory protein-like) was identified from patients suffering from nonspecific X-linked mental retardation (MRX) (7). Non-overlapping deletions as well as a nonsense mutation in the intracellular domain of this receptor are found in MRX patients. The exact function of this receptor is currently not known. It is highly expressed in postnatal brain structures that are important for memory and learning. IL1RAPL was independently characterized from homology searches in the Expressed Sequence Tag (EST) data base, which also found TIGIRR (three immunoglobulin domain-containing IL-1 receptor-related) as its close homolog (8). The TIR domains of IL-RAPL and TIGIRR share $72 \%$ amino acid sequence identity (Fig. 1). Like the other IL-1Rs, these two receptors contain a TIR domain in their intracellular region. However, they also contain a 130-residue segment C-terminal to the TIR domain, which is absent in most of the other TLRS and IL-1Rs. Recent studies suggest that residues in this Cterminal segment of IL-1RAPL may interact with neuronal calcium sensor-1 (NCS-1), and IL-1RAPL may have a role in regulating exocytosis of secretory and neurotransmitter substances (9). NCS-1 is up-regulated in schizophrenic and bipolar patients (10).

We have recently reported the crystal structures of the TIR domains of human TLR1 and TLR2 $(11,12)$. The structures 


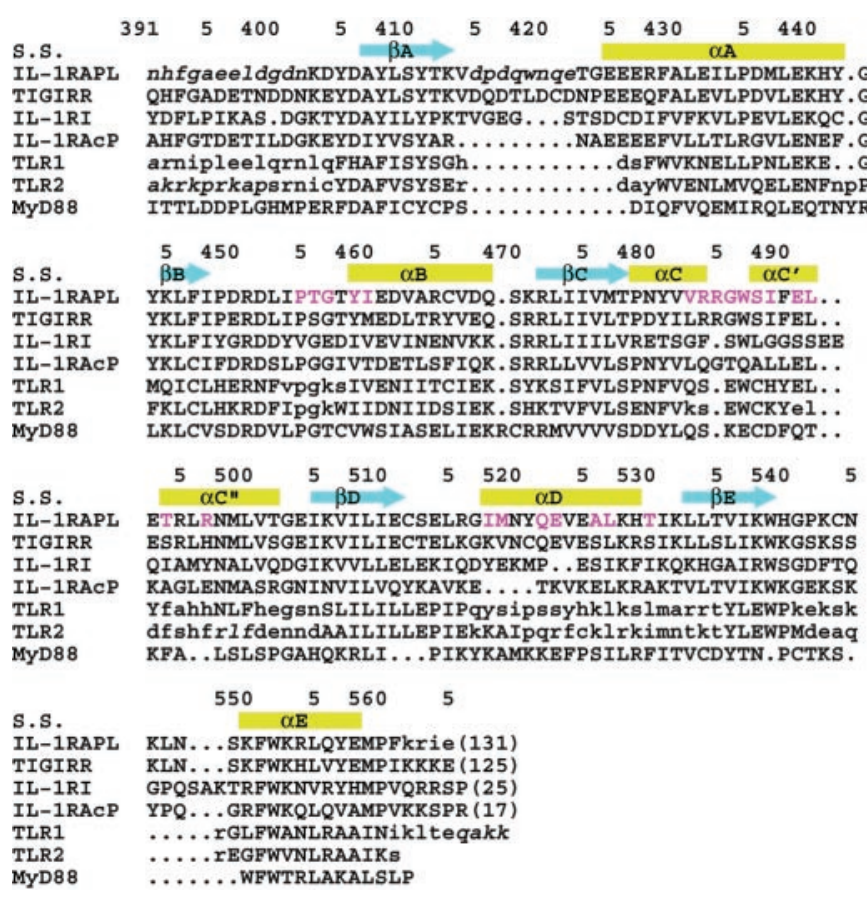

FIG. 1. Sequence alignment of TIR domains. Amino acid sequences of representative TIR domains are shown. The alignment between IL-1RAPL, TLR1, and TLR2 is based on their structures. Residues shown in lowercase in TLR1 and TLR2 have a greater than $3-\AA$ deviation from their equivalents in IL-1RAPL. Residues shown in italic lowercase are not present in the expression constructs. Residues in the dimer interface of the IL-1RAPL TIR domain are shown in magenta.

contain a central five-stranded fully parallel $\beta$-sheet, which is surrounded by $\alpha$-helices on both faces. A loop in the structure, known as the BB loop, forms a prominent feature on the surface of the domain and corresponds to a region of significant sequence conservation among the TIR domains (Fig. 1). The $\mathrm{Lps}^{\mathrm{d}}$ mutation of TLR4 is located at the very tip of this loop, confirming the functional importance of the BB loop. The TIR domains of TLR 1 and TLR2 share $50 \%$ sequence identity, but there are clear structural differences between them. The sequences of the TIR domains of the IL-1Rs are significantly more divergent from those of TLR1 and TLR2 (Fig. 1). We report here the crystal structure of the TIR domain of human IL-1RAPL at 2.3- $\AA$ resolution, the first structure from the IL-1R superfamily. The TIR domain has the same overall fold, but there are significant differences to the structures of the TIR domain of the TLRs. The crystal structure also reveals a dimeric association of TIR domains, which may be important for the functions of this receptor.

\section{MATERIALS AND METHODS}

Protein Expression, Purification, and Crystallization-The TIR domain of human IL-1RAPL (residues 403-563) was subcloned into the pET26b vector (Novagen) and overexpressed in Escherichia coli at $20{ }^{\circ} \mathrm{C}$. The recombinant protein contains a hexahistidine tag at the $\mathrm{C}$ terminus. After cell lysis, the soluble protein was purified by nickelagarose affinity chromatography and anion exchange and gel filtration chromatography. The protein was concentrated to $10 \mathrm{mg} / \mathrm{ml}$ in a buffer containing $25 \mathrm{~mm}$ Tris (pH 7.5), $200 \mathrm{~mm} \mathrm{NaCl}, 5 \mathrm{~mm}$ dithiothreitol, and $5 \%(\mathrm{v} / \mathrm{v})$ glycerol and stored at $-80{ }^{\circ} \mathrm{C}$. The C-terminal His tag was not removed for crystallization.

For the production of selenomethionyl proteins, the expression construct was transformed into the methionine auxotroph $E$. coli DL41(DE3) cells. Bacterial growth was carried out in defined LeMaster media (13), and the protein was purified using the same protocol as for the wild-type protein.

Protein Crystallization-Crystals of the TIR domain were prepared by the vapor diffusion method at $4{ }^{\circ} \mathrm{C}$. Initial crystallization conditions
TABLE I

Summary of crystallographic information

$\begin{array}{lc}\text { Maximum resolution }(\AA) & 2.3 \\ \text { Number of observations } & 150,158 \\ R_{\text {merge }}(\%)^{a} & 5.4(19.7) \\ \text { Figure of merit from MAD phasing } & 0.60 \\ \text { Resolution range for refinement } & 29-2.3 \\ \text { Number of reflections } & 41,323 \\ \text { Completeness }(\%) & 95(82) \\ R \text { factor }{ }^{b}(\%) & 22.9(26.8) \\ \text { Free } R \text { factor }(\%) & 27.3(32.0) \\ \text { r.m.s. deviation in bond lengths }(\AA) & 0.006 \\ \text { r.m.s. deviation in bond angles }\left({ }^{\circ}\right) & 1.3\end{array}$

${ }^{a} R_{\text {merge }}=\Sigma_{h} \sum_{i}\left|I_{h i}-\left\langle I_{h}\right\rangle\right| / \Sigma_{h} \sum_{i} I_{h i}$. The numbers in parentheses are for the highest resolution shell.

${ }^{b} R=\Sigma_{h}\left|F_{h}^{o}-F_{h}^{c}\right| / \Sigma_{h} F_{h}^{o}$.

for the protein were identified by sparse matrix screening with commercial kits (Hampton Research). The reservoir solution contained 100 mM sodium acetate ( $\mathrm{pH} 4.6), 8 \%(\mathrm{w} / \mathrm{v})$ polyethylene glycol 4000, $5 \%(\mathrm{v} / \mathrm{v})$ ethylene glycol, and $5 \mathrm{~mm}$ dithiothreitol. The crystals were cryo-protected with the reservoir solution supplemented with $35 \%(\mathrm{v} / \mathrm{v})$ ethylene glycol and flash-frozen in liquid propane for data collection at $100 \mathrm{~K}$.

Data Collection and Processing-A selenomethionyl multiwavelength anomalous diffraction (MAD) data set to $2.3-\AA$ resolution was collected on an ADSC CCD at the X4A beamline at Brookhaven National Laboratory. Three wavelengths were used: $0.9793 \AA$ (edge), $0.9792 \AA$ (peak), and $0.9200 \AA$ (high energy remote). The diffraction images were processed and scaled with the HKL package (14). The crystal belongs to the space group $\mathrm{P} 2{ }_{1} 2_{1} 2_{1}$, with unit cell parameters of $a=52.0 \AA, b=53.3 \AA$, and $c=183.2 \AA$. There are two molecules in the asymmetric unit, giving a $V_{\max }$ of $3.1 \AA^{3} /$ dalton. The data processing statistics are summarized in Table I.

Structure Determination and Refinement-The locations of 9 selenium atoms were determined with the MAD data using the program SOLVE (15), which also phased all the reflections up to 2.3-Å resolution. After phase improvement by solvent flattening, automatic chain tracing located $70 \%$ of the residues in the two molecules. Additional residues were manually built into the electron density with the program $\mathrm{O}$ (16). The structure refinement was carried out with the program CNS (17). The statistics on the structure refinement are summarized in Table I.

Reporter Gene Assays-HEK293 were seeded $\left(10^{5}\right.$ cells $\left./ \mathrm{ml}\right)$ onto 96 well plates $24 \mathrm{~h}$ before transfection with $80 \mathrm{ng}$ of Gal-luciferase (PathDetect trans-reporting system, Stratagene), $40 \mathrm{ng}$ of Renilla luciferase, $2 \mathrm{ng}$ of c-jun, and the indicated amount of IL-1RAPL- or TIGIRRexpressing plasmid (220 ng total) using Genejuice (Novagen, Madison, WI) according to the manufacturer's recommendations. The amount of DNA transfected was kept constant by the addition of various amounts of the appropriate empty vector plasmid. $16 \mathrm{~h}$ post-transfection, cells were lysed for $15 \mathrm{~min}$ at room temperature with $50 \mu \mathrm{l}$ of passive lysis buffer (Promega, Southampton, United Kingdom). After this, $50 \%$ of the supernatant was used to determine firefly luciferase activity, and an equivalent amount was used for Renilla luciferase activity. Firefly and Renilla luciferase activity was assayed using standard protocols. Levels of firefly luciferase expression were normalized against Renilla activity as a control for transfection efficiency and expressed as -fold stimulation over the unstimulated empty vector control. The deletion mutants of IL-1RAPL were created by introducing stop codons at desired positions in the gene with the QuikChange kit (Stratagene).

\section{RESULTS AND DISCUSSION}

The Structure Determination-The crystal structure of the Toll/interleukin-1 receptor (TIR) domain of human IL-1RAPL has been determined at $2.3-\AA$ resolution by the selenomethionyl MAD method (Table I) (18). The positions of the selenium atoms as well as the phases of all observed reflections were determined with the MAD data (15), which allowed the automatic placement of roughly $70 \%$ of the residues of the two molecules in the asymmetric unit. The current atomic model has good agreement with the observed x-ray diffraction data, with an $R$ factor of $22.9 \%$. The root mean square (r.m.s.) deviation in bond lengths is $0.006 \AA$, and that in bond angles is $1.2^{\circ}$. A total of $89.6 \%$ of the residues is in the most favored regions, and the remaining $10.4 \%$ of the residues is in the additional allowed regions of the Ramachandran plot. 
FIG. 2. Structure of the TIR domain of human IL-1RAPL. $A$, schematic drawing of the structure of the TIR domain. The $\beta$-strands are shown in cyan, $\alpha$ helices in yellow and the connecting loops in magenta. $B$, overlay of the structure of the TIR domains of IL-1RAPL (yellow), and TLR1 (cyan). Produced with Ribbons (20).

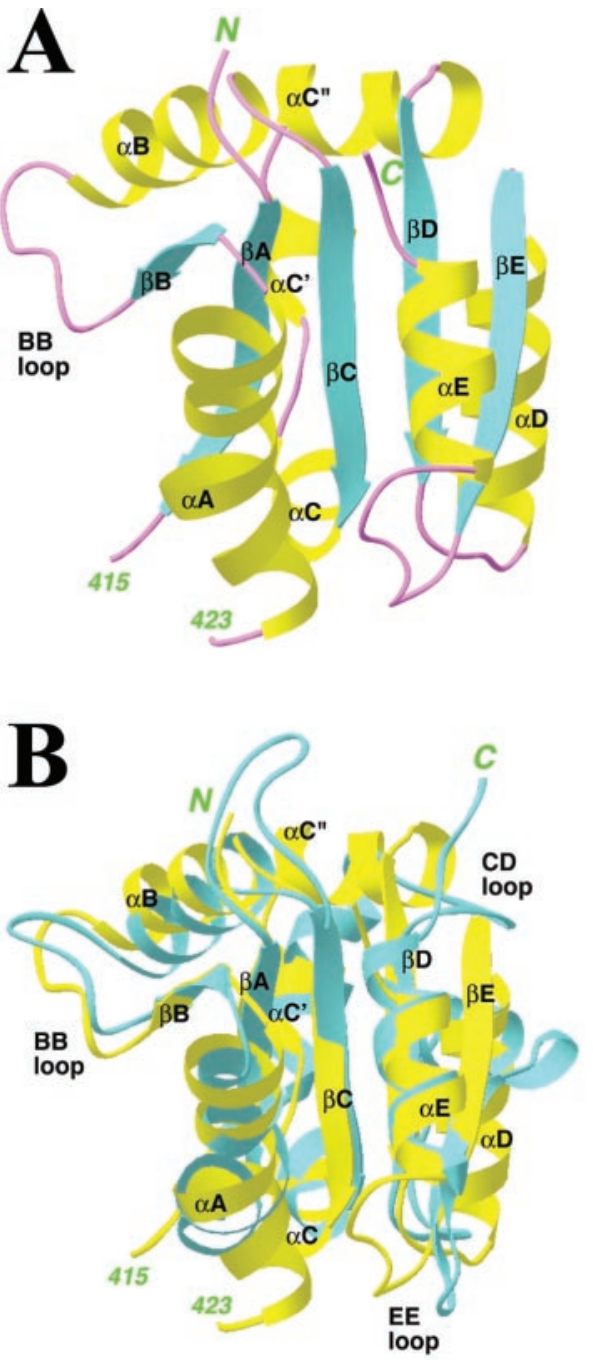

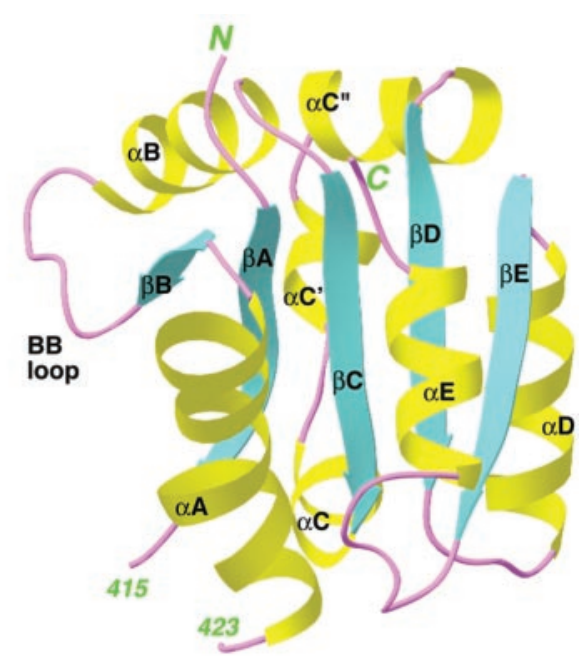

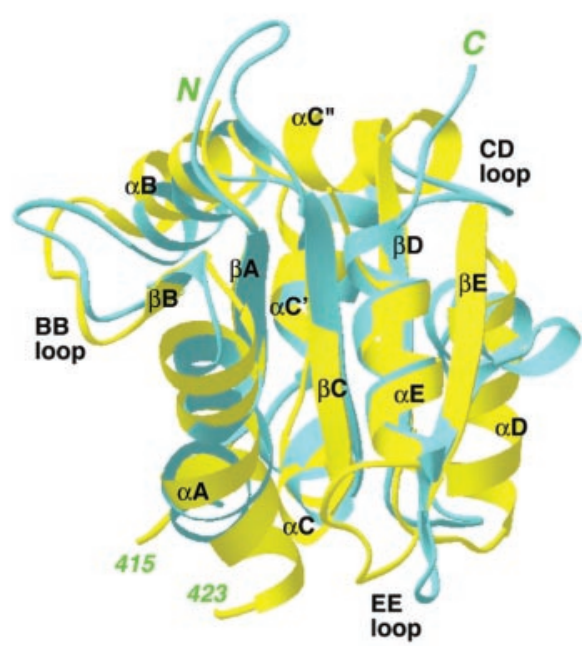

The expression construct covers residues 403-563 of IL1RAPL, which misses the first 20 residues of the intracellular region of the receptor as well as the 130-residue segment at the $\mathrm{C}$ terminus that is present in this receptor and TIGIRR. The current atomic model contains residues 403-415, residues 423-561 for one molecule, and residues 403-414 and 423-561 for the other molecule in the crystallographic asymmetric unit. The atomic coordinates have been deposited at the Protein Data Bank (accession code 1T3G).

Large Conformational Differences to the Structures of TLR TIR Domains - This is the first structure of a TIR domain from the IL-1R superfamily of receptors. The structure of the TIR domain of human IL-1RAPL contains a central five-stranded fully parallel $\beta$-sheet, which is surrounded by helices on both sides (Fig. 2A). The overall backbone fold of this structure is the same as that of the TIR domain of human TLR1 and TLR2 that we reported earlier (11). The r.m.s. distance for 99 equivalent C- $\alpha$ atoms between the TIR domains of IL-1RAPL and TLR1 is $1.4 \AA$, with the residues in the $\beta$-sheet having the highest structural conservation (Fig. $2 B$ ).

However, there are significant differences in the conformations of the TIR domains of TLR 1 and IL-1RAPL. This supports the idea that the TIR domains have large degrees of conformational variability, which may be important for ensuring specificity in signal transduction by these receptors (11). For the $\beta$-sheet, strand $\beta \mathrm{E}$ in IL-1RAPL is longer at the N-terminal end than strand $\beta \mathrm{E}$ in TLR1 (Fig. $2 B$ ). The largest differences between the two structures are seen in some of their helices. On one face of the $\beta$-sheet, the axis of helix $\alpha \mathrm{D}$ in IL-1RAPL is oriented almost perpendicularly to that of the $\alpha$ D helix in TLR1 and TLR2 (Fig. 2B). On the other face of the $\beta$-sheet, the beginning of the $\alpha \mathrm{A}$ helix has different conformations in the two structures. This may be related to the fact that IL-1RAPL has an insertion between $\beta \mathrm{A}$ and $\alpha \mathrm{A}$ (Fig. 1). Although most of the residues in this inserted segment are disordered in the current structure, they may have affected the positioning of the beginning of the $\alpha \mathrm{A}$ helix. Structural differences are also observed for the CD and EE loops between the two TIR domains (Fig. 2B).

Another structural difference between IL-1RAPL and TLR1 is in the crossover from $\beta C$ to $\beta D$. In the TIR domain of TLR1 and TLR2, two short helices ( $\alpha \mathrm{C}$ and $\alpha \mathrm{C}^{\prime}$ ) were observed, followed by a highly disordered region. In IL-1RAPL, however, the two short helices are followed by a third, well ordered helix $\left(\alpha \mathrm{C}^{\prime}\right.$ ) (Fig. 2A). The conformations of the $\alpha \mathrm{C}$ and $\alpha \mathrm{C}^{\prime}$ helices are also different between IL-1RAPL and TLR1 (Fig. 2B). The observed structural differences may affect how these TIR domains form homo- or hetero-oligomers during signal transduction (see below).

The TIR domains from the IL-1Rs contain a highly conserved DGK motif at its $\mathrm{N}$ terminus (Fig. 1), with the Lys residue corresponding to the first residue (403) in our expression construct for IL-1RAPL. This Lys residue is fully exposed to the solvent and has weak electron density in our structure. To 
FIG. 3. Conformation of the BB loop. Overlay of the structure of the $\mathrm{BB}$ loop of IL-1RAPL (yellow) and TLR1 (cyan). Produced with Ribbons (20).

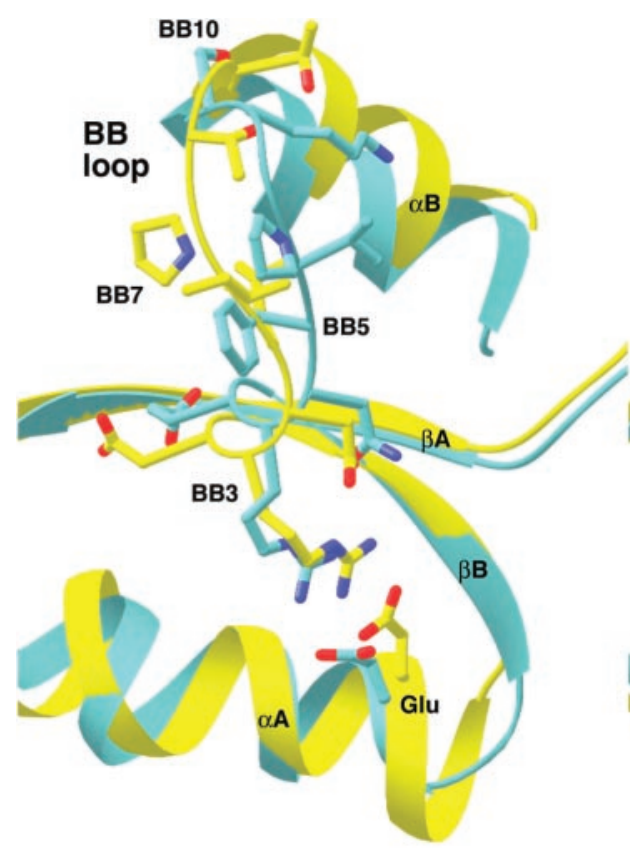

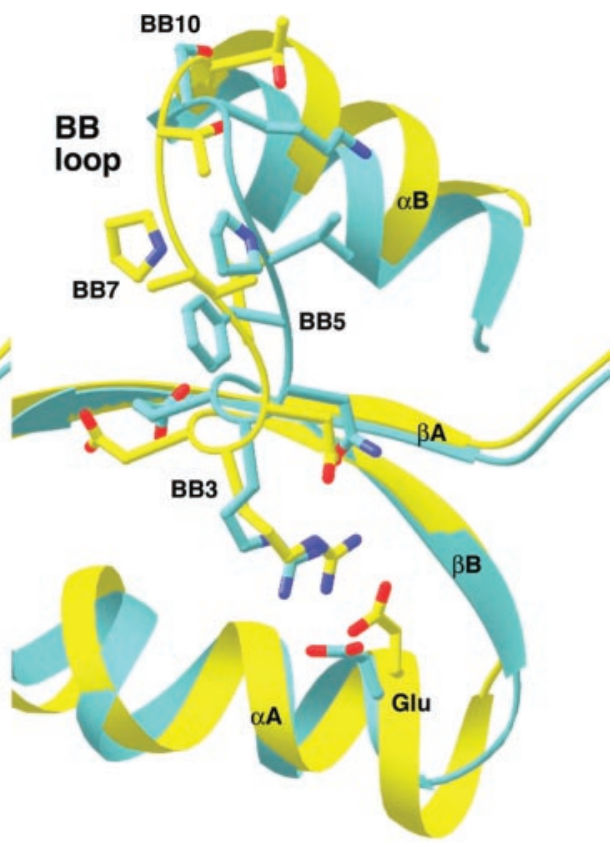

determine the conformation of this entire segment, we produced additional IL-1RAPL constructs, extending the $\mathrm{N}$ terminus. One of these constructs (covering residues 395-584) produced soluble protein, which enabled the preparation of small, weakly diffracting crystals. An x-ray diffraction data set to $2.3-\AA$ resolution was collected, and the subsequent crystallographic analysis showed that the DGK motif at the $\mathrm{N}$ terminus is disordered in this longer construct. This construct also contains 20 additional residues at the $\mathrm{C}$ terminus, but they are disordered in the structure as well.

Conformational Differences in the BB Loop-The BB loop contains about 10 residues and links strand $\beta \mathrm{B}$ and helix $\alpha \mathrm{B}$ in the structures of TIR domains (11). Several residues in this loop are highly conserved among the TIR domains, including an Arg residue at the third position (the BB3 residue), Asp at BB4, Pro at BB7 (the Lps ${ }^{\mathrm{d}}$ mutation site), and Gly at BB8. These residues are in a motif $R D \Phi \Phi P G$, where $\Phi$ is generally a hydrophobic residue in the TIR domains.

The structures of the TIR domains of TLR1 and TLR2 showed that the Arg residue at the BB3 position is involved in an ion pair interaction with a conserved Glu residue near the end of helix $\alpha \mathrm{A}$ ( $\alpha \mathrm{A} 13$ residue). This interaction is also observed in the TIR domain of IL-1RAPL, and the BB4 Asp residue also pairs ions with the BB3 Arg residue (Fig. 3).

Farther down the BB loop, large differences are observed both in the positions of the main chain and the conformations of the side chain between the TIR domains of IL-1RAPL and TLR1 (Fig. 3). The position of helix $\alpha \mathrm{B}$ is also different between the two structures. The side chain of the BB5 residue is pointed into the solvent in the TLR1 TIR domain, whereas it is buried in the IL-1RAPL structure. The positions of the BB7 residues in the two structures are separated by a distance of about $5 \AA$. At the BB8 position, IL-1RAPL has a Thr residue, whereas most other TIR domains have a Gly (Fig. 1). The side chain of this Thr residue lies on the surface of the domain, hydrogenbonded to the side chain of the Thr residue at the BB10 position (Fig. 3), which is unique to IL-1RAPL and TIGIRR (Fig. 1).

A Dimer of the TIR Domain of IL-1RAPL in the CrystalBiological studies suggest that TIR domains may function through homotypic interactions. Upon receptor activation the TIR domains of the receptors may form a complex that can in turn recruit the downstream adapter molecules (MyD88, MAL, and TRIF) through interactions among their TIR domains. Our studies with purified TIR domains, however, showed that the intrinsic affinity of the TIR domains for self-association is generally rather low. Most of the TIR domains that we have purified behave as monomers in solution, even at $1 \mathrm{mg} / \mathrm{ml}(50 \mu \mathrm{M})$ concentration. Gel filtration and light scattering studies showed that the TIR domain of IL-1RAPL is also monomeric in solution. ${ }^{2}$

In the crystal of the TIR domain of IL-1RAPL, there are two molecules in the asymmetric unit, related by a non-crystallographic 2-fold symmetry axis (Fig. $4 A$ ). Unexpectedly, the two monomers share an extensive contact interface in this dimer, with each monomer contributing about $1000 \AA^{2}$ of surface area. The two monomers are arranged such that the helices on one face of the $\beta$-sheet $\left(\alpha \mathrm{B}, \alpha \mathrm{C}, \alpha \mathrm{C}^{\prime}, \alpha \mathrm{C}^{\prime}\right.$, and $\left.\alpha \mathrm{D}\right)$ in one monomer interact with their equivalents in the other monomer, whereas helices on the other face ( $\alpha \mathrm{A}$ and $\alpha \mathrm{E}$ ) are not involved in this interface (Fig. 1).

At the interior of this interface is the contact among helices $\alpha \mathrm{C}, \alpha \mathrm{C}^{\prime}$, and $\alpha \mathrm{C}^{\prime}$ in one monomer with their equivalents in the other (Fig. $4 B$ ). Leu-492 in helix $\alpha \mathrm{C}^{\prime}$ is completely shielded from the solvent by this contact, contributing $125 \AA^{2}$ of buried surface area. The side chain of this residue is pointed toward the $\alpha \mathrm{D}$ helix in the other monomer, interacting with Ala-526' and Thr-530' (primed residue numbers indicate the other monomer), as well as Trp-487' in the loop between helices $\alpha \mathrm{C}$ and $\alpha \mathrm{C}^{\prime}$. Three Arg residues, 484, 485, and 497, are located next to the 2 -fold axis of the dimer and contribute $195 \AA^{2}$ total to the surface area burial. In addition, Arg-485 and Arg-497 are involved in ion pair interactions with residues Glu-523 and Glu491 , respectively, from the same monomer of the dimer.

The BB loops of the two monomers are located at the edges of the dimer interface. Together with residues in helix $\alpha \mathrm{B}$, they contact the $\alpha \mathrm{D}$ helix of the other monomer, contributing $165 \AA^{2}$ total to the surface area burial.

This TIR Domain Dimer May Be Unique to IL-1RAPL-The detailed interactions between the two monomers in the dimer of IL-1RAPL appear to be unique to this receptor and possibly its close homolog TIGIRR, as many of the residues in this interface are conserved only in these two receptors (Fig. 1). For

\footnotetext{
${ }^{2}$ J. A. Khan and L. Tong, unpublished data.
} 
FIG. 4. Dimer of the TIR domain of IL-1RAPL. $A$, stereo diagram showing the dimer of the TIR domain, viewed down the 2 -fold axis. The two monomers are colored in green and cyan, respectively. $B$, detailed interactions in the dimer interface of the TIR domain of IL1RAPL. The 2-fold axis of the dimer is indicated with the black bar. Produced with Ribbons (20).
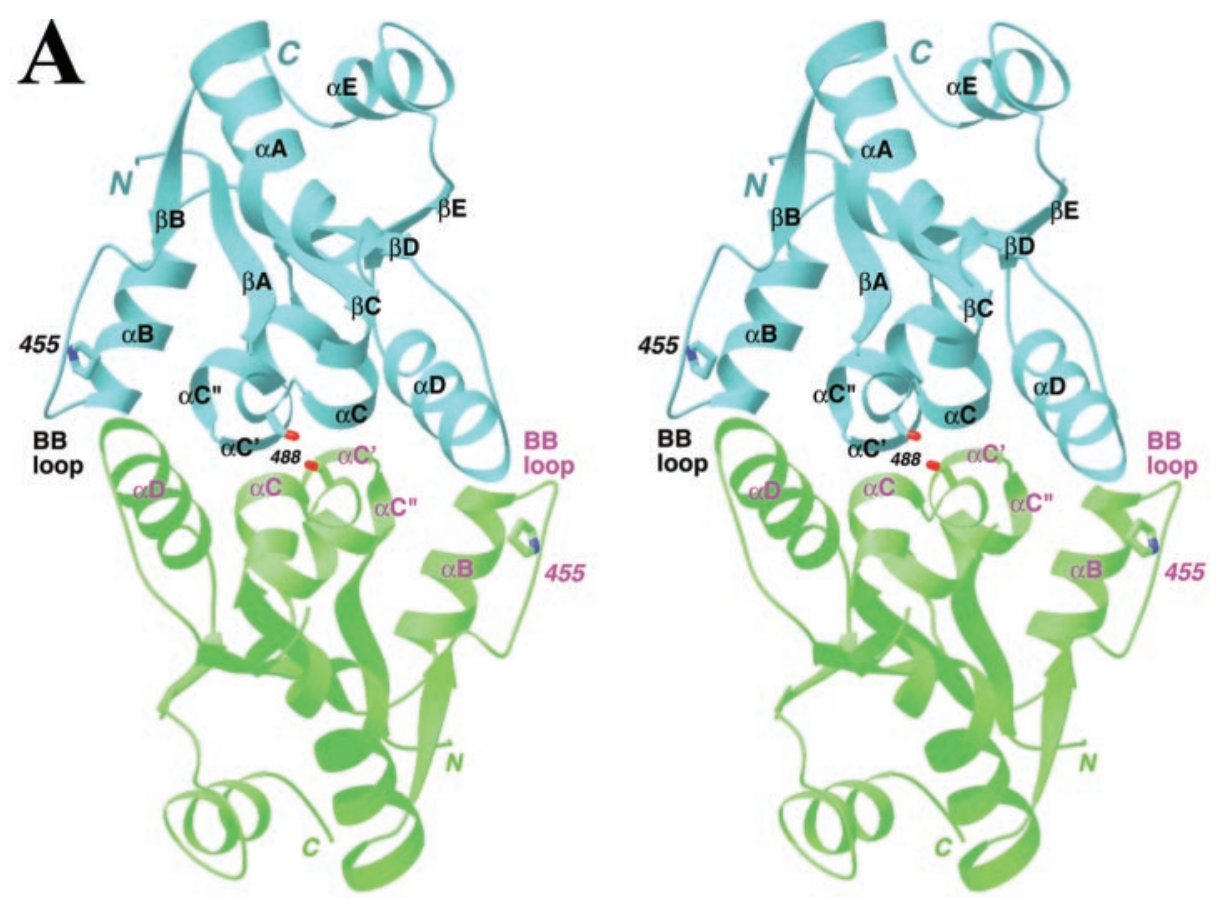

B

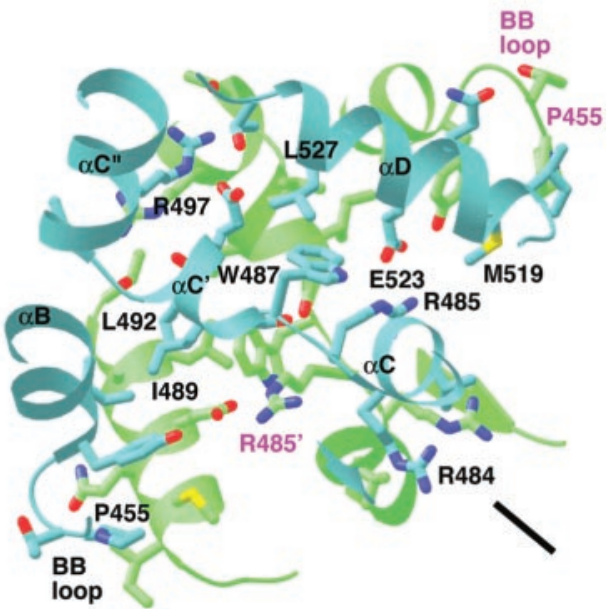

example, none of the three Arg residues $(484,485,497)$ in the IL-1RAPL dimer interface is conserved in the other TIR domains (Fig. 1). In addition Glu-523, the ion pair partner for Arg-485, is not conserved. This unique dimer interface is consistent with the distinct functional properties of these two receptors (see below) (8).

At the center of the dimer interface is the Ser- 488 residue in helix $\alpha \mathrm{C}^{\prime}$ (Fig. 4A). It is situated right next to the 2 -fold axis of the dimer such that it forms a tight hydrogen bond with its equivalent, Ser-488', in the other monomer. Interestingly, a close contact for the equivalent residue of Ser-488 in TLR1, Cys-707, was observed in the crystal structure of that TIR domain (11). As a matter of fact, Cys-707 forms a disulfide bond with Cys-707' in the other monomer of a dimer (Fig. 5). As a result of this disulfide link, the observed dimer interface in the TIR domain of TLR1 was thought to be biologically irrelevant $(11,12)$. However, the current structure of the IL-1RAPL TIR domain suggests that close contact of this residue in the dimer interface could be functionally relevant. This is supported by our observation that mutation of the equivalent Cys residue in TLR2 abolished receptor function (12).

The dimers of the TIR domains of IL-1RAPL and TLR1 are rather different. This is likely due in large part to the significant change in the conformation of the $\alpha \mathrm{D}$ helix between the two TIR domains. The dimer association observed for IL1RAPL is impossible for the TLR1 TIR domain as it would cause steric clashes for the $\alpha \mathrm{D}$ helix. A smaller surface area, about $600 \AA^{2}$ for each monomer, is buried by the dimer of the TIR domain of TLR1 (Fig. 5).

Functional Studies with IL-1RAPL-To assess the biological relevance of the structural information, we attempted functional studies with the IL-1RAPL receptor. Of special interest is the functional role of the dimer that is observed in the crystal. This can only be assessed using IL-1RAPL and possibly its close homolog TIGIRR as the dimer may not be present for the other receptors.

Earlier studies have shown that IL-1RAPL and TIGIRR cannot activate the $\mathrm{NF}_{\kappa} \mathrm{B}$ transcription factor (8). Recent studies with another IL-1R family member, T1/ST2, showed that it can activate the MAP kinases ERK, p38, and JNK, even though it cannot activate $\mathrm{NF}_{\kappa} \mathrm{B}$ (19). This led us to examine whether IL-1RAPL can also activate the MAP kinases. Our experimental data showed that this receptor can activate JNK (Fig. 6) but not the ERK and p38 MAP kinases, and our data also confirm that IL-1RAPL cannot activate $\mathrm{NF}_{\kappa} \mathrm{B}$ under the conditions used (data not shown). It is possible that in the system studied here, a co-receptor not expressed in these cells may be needed for IL-1RAPL to activate $\mathrm{NF}_{\kappa} \mathrm{B}$. 
FIG. 5. Dimer of the TIR domain of TLR1. Stereo diagram showing the dimer of the TIR domain, viewed down the 2 -fold axis. The two monomers are colored in green and cyan, respectively. The disulfide link between Cys-707 and Cys-707' is shown. Produced with Ribbons (20).
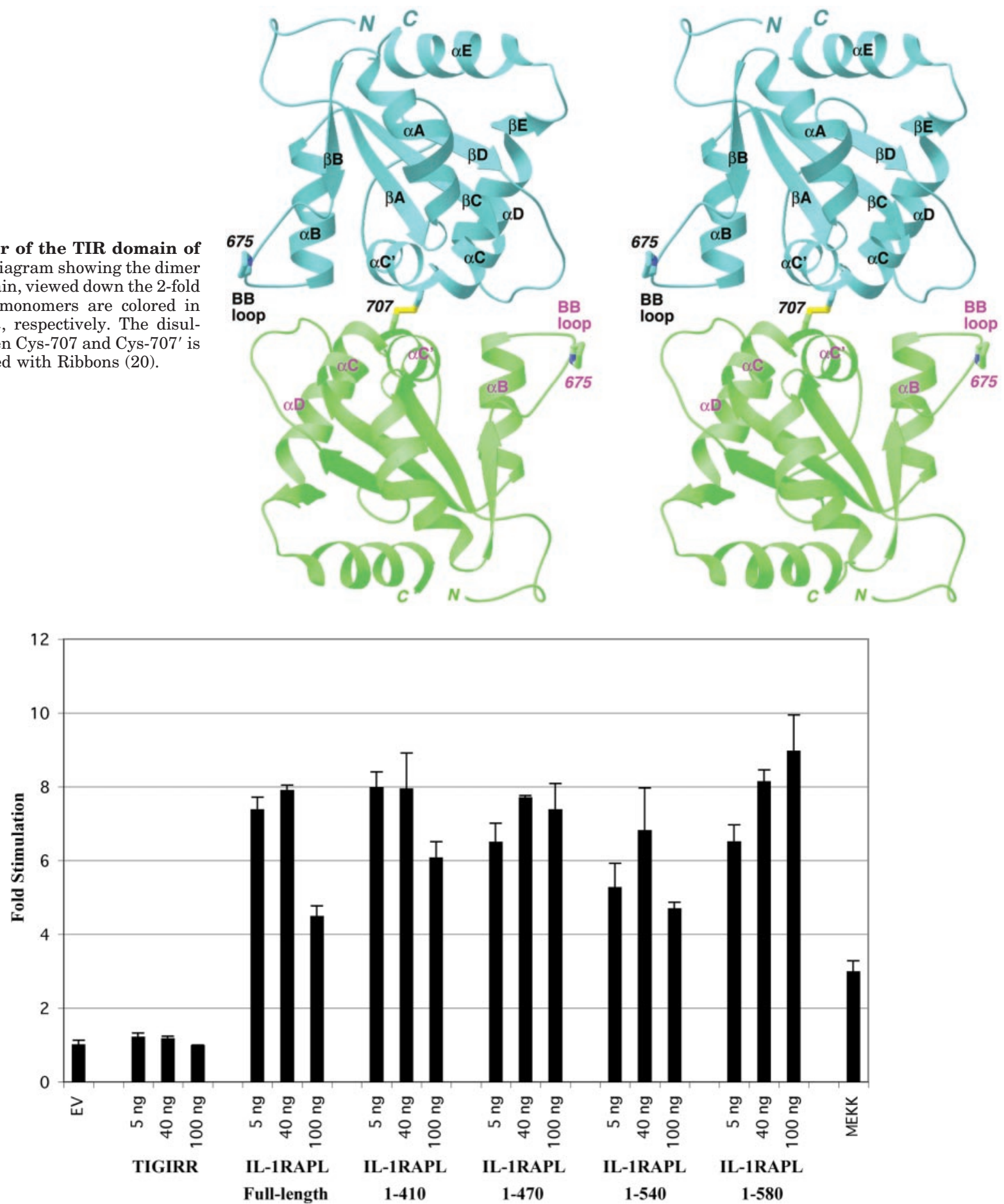

FIG. 6. IL-1RAPL activates JNK. HEK293 cells were transfected with the indicated amounts of plasmid encoding TIGIRR, IL-1RAPL, and MEKK along with Gal-luciferase, c-jun, and thymidine kinase Renilla luciferase reporter gene constructs. The deletion mutants of IL-1RAPL are truncated from the $\mathrm{C}$ terminus. Extracts were prepared and measured for luciferase activity. Results are normalized for Renilla luciferase activity and represented as -fold stimulation over the nonstimulated EV control. Results are expressed as mean \pm S.D. from three separate experiments, each carried out in triplicate. $E V$, empty vector.

Further studies of this activation by IL-1RAPL suggest that it is independent of the TIR domain. Truncation mutants missing part of or even the entire TIR domain were found to be able to activate JNK to the same extent as the full-length receptor (Fig. 6). It is unlikely that this activation is an artifact, as TIGIRR cannot activate this signal, despite the strong sequence conservation. Moreover, the -fold stimulation by IL1RAPL is more than twice that produced by MEKK (Fig. 6). IL-1RAPL may be able to activate JNK by recruiting other molecules through its extracellular domain where the sequence homology with TIGIRR is weaker. All the deletion mutants studied here contain a small intracellular segment just prior to the TIR domain (residues 379-403). However, it is probably unlikely for this segment to mediate the activation of JNK, as it shares significant homology with TIGIRR. Further studies are needed to assess the physiological relevance of this JNK activation by IL-1RAPL.

In summary, we have determined the crystal structure of the TIR domain of human IL-1RAPL at 2.3- $\AA$ resolution. As the first structure of the TIR domain from the IL-1R superfamily of 
receptors, it shows significant differences to the structures of the TIR domains of TLR1 and TLR2 that we reported earlier. This is consistent with the idea that sequence and structural diversity among these domains is important for specificity in the signal transduction process. The unique structural features of the TIR domain in IL-1RAPL when compared with TLR 1 and TLR2 may be the molecular basis for its inability to activate $\mathrm{NF} \kappa \mathrm{B}$. The structural analyses reveal the presence of a dimer with a rather extensive interface in the crystals of this domain. The detailed interactions in this dimer appear to be unique to IL-1RAPL and possibly its close homolog TIGIRR, and therefore they cannot be studied using IL-1RI and IL-1RAcP. The assessment of the functional relevance of the observed dimer will have to await the development of a reporter assay for these two receptors.

Structural observations of the TIR domains of IL-1RAPL and TLR1 suggest that residues in the $\alpha \mathrm{C}^{\prime}$ helix, especially Ser-488/ Cys-707, might be important for the function of TIR domains. This is supported by our studies showing that mutation of the equivalent Cys-713 residue in TLR2, even to Ser, abolished the function of the receptor (12). Sequence comparison among the TIR domains show that this residue is almost strictly conserved as Cys among the mammalian TLRs, but it is not conserved among the IL-1Rs and the adapter TIR domains (Fig. 1). The molecular mechanism of the functional requirement of a Cys residue at this position in the TLRs remains to be established.

Acknowledgments-We thank Randy Abramowitz and Xiaochun Yang for setting up the X4A beamline, Gerwald Jogl, Hailong Zhang, and Xiao Tao for help with data collection at the synchrotron, and John Sims for providing the IL-1RAPL and TIGIRR plasmids and for helpful discussions.

\section{REFERENCES}

1. Dunne, A., and O'Neill, L. A. J. (2003) Science's STKE http://stke. sciencemag.org/cgi/content/full/sigtrans;2003/171/re3

2. Medzhitov, R., and Janeway, C. A., Jr. (1997) Cell 91, 295-298

3. Anderson, K. V. (2000) Curr. Opin. Immunol. 12, 13-19

4. Sims, J. E. (2002) Curr. Opin. Immunol. 14, 117-122

5. O'Neill, L. A. J., Fitzgerald, K. A., and Bowie, A. G. (2003) Trends Immunol. 24, 286-289

6. Poltorak, A., He, X., Smirnova, I., Liu, M.-Y., Huffel, C. V., Du, X., Birdwell, D., Alejos, E., Silva, M., Galanos, C., Freudenberg, M., Ricciardi-Castagnoli, P., Layton, B., and Beutler, B. (1998) Science 282, 2085-2088

7. Carrie, A., Jun, L., Bienvenu, T., Vinet, M.-C., McDonell, N., Couvert, P. Zemni, R., Cardona, A., Buggenhout, G. V., Frints, S., Hamel, B., Moraine, C., Ropers, H. H., Strom, T., Howell, G. R., Whittaker, A., Ross, M. T., Kahn, A., Fryns, J.-P., Beldjord, C., Marynen, P., and Chelly, J. (1999) Nat. Genet. 23, 25-31

8. Born, T. L., Smith, D. E., Garka, K. E., Renshaw, B. R., Bertles, J. S., and Sims, J. E. (2000) J. Biol. Chem. 275, 29946-29954

9. Bahi, N., Friocourt, G., Carrie, A., Graham, M. E., Weiss, J. L., Chafey, P., Fauchereau, F., Burgoyne, R. D., and Chelly, J. (2003) Hum. Mol. Genet. 12, $1415-1425$

10. Koh, P. O., Undie, A. S., Kabbani, N., Levenson, R., Goldman-Rakic, P. S., and Lidow, M. S. (2003) Proc. Natl. Acad. Sci. U. S. A. 100, 313-317

11. Xu, Y., Tao, X., Shen, B., Horng, T., Medzhitov, R., Manley, J. L., and Tong, L. (2000) Nature 408, 111-115

12. Tao, X., Xu, Y., Zheng, Y., Beg, A., and Tong, L. (2002) Biochem. Biophys. Res. Commun. 299, 216-221

13. Hendrickson, W. A., Horton, J. R., and LeMaster, D. M. (1990) EMBO J. 9 , $1665-1672$

14. Otwinowski, Z., and Minor, W. (1997) Methods Enzymol. 276, 307-326

15. Terwilliger, T. C., and Berendzen, J. (1999) Acta Crystallogr. Sect. D Biol. Crystallogr. 55, 1872-1877

16. Jones, T. A., Zou, J. Y., Cowan, S. W., and Kjeldgaard, M. (1991) Acta Crystallogr. Sect. A 47, 110-119

17. Brunger, A. T., Adams, P. D., Clore, G. M., DeLano, W. L., Gros, P., GrosseKunstleve, R. W., Jiang, J.-S., Kuszewski, J., Nilges, M., Pannu, N. S., Read, R. J., Rice, L. M., Simonson, T., and Warren, G. L. (1998) Acta Crystallogr. Sect. D Biol. Crystallogr. 54, 905-921

18. Hendrickson, W. A. (1991) Science 254, 51-58

19. Brint, E. K., Fitzgerald, K. A., Smith, P., Coyle, A. J., Gutierrez-Ramos, J.-C., Fallon, P. G., and O'Neill, L. A. J. (2002) J. Biol. Chem. 277, 49205-49211

20. Carson, M. (1987) J. Mol. Graphics 5, 103-106 